\title{
From Photodriven Charge Accumulation to Fueling Enzyme Cascades in Molecular Factories
}

\author{
Yasunori Okamoto, Thomas R. Ward*, and Oliver S. Wenger*
}

\begin{abstract}
In a multi-disciplinary team effort we gather experts on light-to-chemical energy conversion, artificial metalloenzymes, and bio-inspired polymer vesicles in order to construct molecular factories which produce added-value chemicals in an overall process fueled by solar energy. We outline our long-term vision and discuss specific challenges associated with this endeavor.
\end{abstract}

Keywords: Molecular factories · Solar energy

\section{Introduction}

Every factory needs energy input. The use of solar energy would be a particularly elegant approach to fueling molecular factories that produce chemicals of added value. Cascades of artificial metalloenzymes and natural enzymes have recently emerged as a promising strategy to address synthetic challenges, ${ }^{[1,2]}$ and in the future such cascades could become the workhorses of molecular factories. Artificial metalloenzymes result from the encapsulation of small organometallic catalysts into a protein pocket which is reasonably well shielded from the environment, and consequently the catalyst is not deactivated easily even in chemically complex systems. ${ }^{[3,4]}$ Many of the enzymes suitable for these cascades require energy input in the form of NADH (nicotinamide adenine dinucleotide) hence our efforts to convert solar energy into chemical fuel will be primarily geared at obtaining NADH or ana$\operatorname{logs}$ thereof. Compartmentalization of the envisioned molecular factories will be of key importance, for example for the spatial separation of oxidation and reduction products in the course of photochemical NADH production, but also in order to avoid compatibility problems and mutual inhibition between individual enzymes and cofactors in the cascade. Thus, our long-term vision is the construction of functional molecular factories with enzyme cascades made from artificial metalloenzymes, fueled by photochemically produced NADH. The products of these molecular factories will be added-value chemicals from an overall process driven by solar energy.

The molecular factories will be constructed as illustrated in Fig. 1: Reducing equivalents from a co-substrate are driven across a membrane to an artificial photosynthetic reaction center which reduces $\mathrm{NAD}^{+}$to NADH under the action of visible light. NADH serves as an energy input to a first artificial metalloenzyme which produces a mediator that subsequently enables a second (artificial) enzyme to convert substrate into product. Ideally, the product is then released from the closed compartment of the molecular factory. This vision requires a team-effort involving experts on light-to-chemical energy conversion, en- zyme cascades, and nanostructures such as bio-inspired polymer vesicles.

\section{Challenges Regarding Photochemical Production of NADH}

Photoinduced electron transfer commonly leads to simple electron-hole pairs. In principle, these redox equivalents can be used in secondary reactions to form reduction and oxidation products in separate compartments. However, many of the most interesting fuel-forming reactions (e.g. the production of solar fuels such as $\mathrm{H}_{2}, \mathrm{H}_{2} \mathrm{O}_{2}$, $\mathrm{CH}_{3} \mathrm{OH}, \mathrm{CH}_{4}$ from $\mathrm{H}_{2} \mathrm{O}$ or $\mathrm{CO}_{2}$ ) inherently require multi-electron transformations (often coupled to proton transfer), which cannot be performed with single electrons. This is no different for NADH which requires two electrons and one proton to be formed from $\mathrm{NAD}^{+}$.

Many current studies on artificial photosynthetic systems focus on one of two half-reactions, either the production of a fuel with sacrificial electron donors or the formation of oxidation products (mostly

${ }^{\star}$ Correspondence: Prof. T. Ward, Prof. O. Wenger E-mail: thomas.ward@unibas.ch,

oliver.wenger@unibas.ch

Department of Chemistry

University of Basel

Spitalstrasse 51 and St. Johanns-Ring 19

$\mathrm{CH}-4056$ Basel

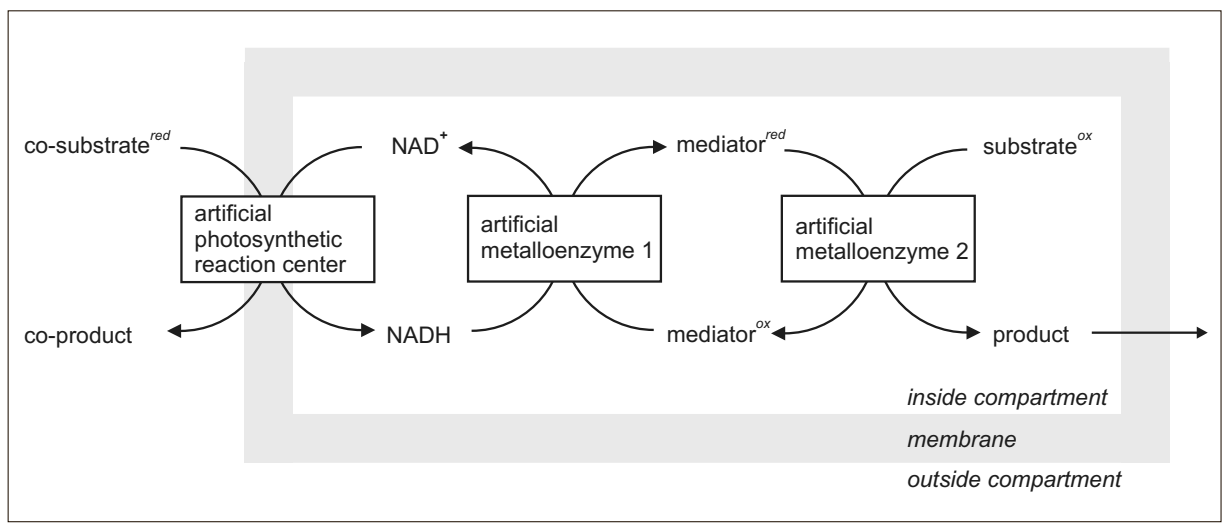

Fig. 1. Illustration of the concept of light-driven enzyme cascades in molecular factories. 
$\mathrm{O}_{2}$ from $\mathrm{H}_{2} \mathrm{O}$ ) using sacrificial electron acceptors. ${ }^{[5-7]}$ However, the use of sacrificial reagents will not permit sustainable lightto-chemical energy conversion.

Against this background, it seems desirable to develop concepts that will allow i) the light-driven accumulation of multiple redox equivalents on a given molecular unit, and ii) the use of these redox equivalents to form a solar fuel. Successful implementation of these two aspects will result in the artificial photosynthetic reaction center shown in Fig. 1. For the purpose of constructing molecular factories, we will mostly rely on sacrificial electron donors for accumulating reduction equivalents and for the formation of NADH from $\mathrm{NAD}^{+}$, while the development of artificial photosynthetic concepts that do not require sacrificial reagents is targeted in separate efforts by some of us. ${ }^{[8,9]}$

So far, we have explored a molecular triad comprised of a central naphthalene diimide (NDI) unit flanked by two peripheral $\mathrm{Ru}(\mathrm{bpy})_{3}{ }^{2+}$ (bpy $=2,2$ '-bipyridine) photosensitizers (Fig. 2). ${ }^{[10]}$ Selective excitation of the ruthenium(II) complexes in neat $\mathrm{CH}_{3} \mathrm{CN}$ rapidly ( $<10 \mathrm{~ns}$ ) leads to intramolecular photoinduced electron transfer to result in $\mathrm{NDI}^{-}$, but the resulting charge-separated state is unstable and decays back to the ground state almost equally rapidly. Charge accumulation on NDI in neat $\mathrm{CH}_{3} \mathrm{CN}$ is not possible in this triad. The situation changes drastically when excess triethylamine is present. In this case triethylamine serves as a sacrificial electron donor, and charge accumulation on NDI becomes readily possible, at least under steady-state irradiation. When illuminating a $10^{-5} \mathrm{M}$ solution of the triad in $\mathrm{CH}_{3} \mathrm{CN}$ containing $0.5 \mathrm{M}$ triethylamine at $410 \mathrm{~nm}$ using a flux of $\sim 3 \times 10^{16}$ photons/s, NDI is converted to $\mathrm{NDI}^{-}$in the course of $40 \mathrm{~s}$ (Fig. 3). Continued irradiation then leads to the formation of $\mathrm{NDI}^{2-}$, and this process reaches completion after $\sim 800$ s. The overall quantum yield for electron accumulation is $\sim 0.02 \%$, yet this experiment represents an important proofof-concept. However, at this point it is unclear yet whether the formation of $\mathrm{NDI}^{2-}$ is the result of the consecutive absorption of two photons by a given triad, or whether it results from a (bimolecular) disproportionation reaction between two $\mathrm{NDI}^{-}$units. Even if the disproportionation equilibrium is unfavorable, constant removal of neutral NDI by reductive quenching could finally transform all triads to the $\mathrm{NDI}^{2-}$ state. This may seem like a mechanistic aspect that is of minor importance for the overall effect of charge accumulation, yet we consider this a crucial point for the envisioned artificial photosynthetic reaction center where we would like to rely on unimolecular charge accumulation processes for practi- cal purposes. In this study we used NDI as an electron accumulation center because the one- and two-electron reduced forms of this particular acceptor are easily distinguishable by UV-Vis spectroscopy, but in the long run we will aim for other twoelectron acceptors.

The artificial photosynthetic reaction center from Fig. 1 could for example be based on Rh(III) catalysts which are photochemically reduced to $\mathrm{Rh}(\mathrm{I})$-hydride species. The latter are known to be able to perform regioselective reduction of $\mathrm{NAD}^{+}$ to NADH even under photocatalytic conditions. ${ }^{[11,12]}$ Initial studies will focus on NADH-model compounds, such as BNAH (1-benzyl-1,4-dihydronicotinamide) or 10-methyl-9,10-dihydroacridine.

\section{Challenges Regarding Enzyme Cascades}

We have recently developed artificial metalloenzymes that can use $\mathrm{NAD}(\mathrm{P}) \mathrm{H}$ as hydride source for a variety of enantiose-

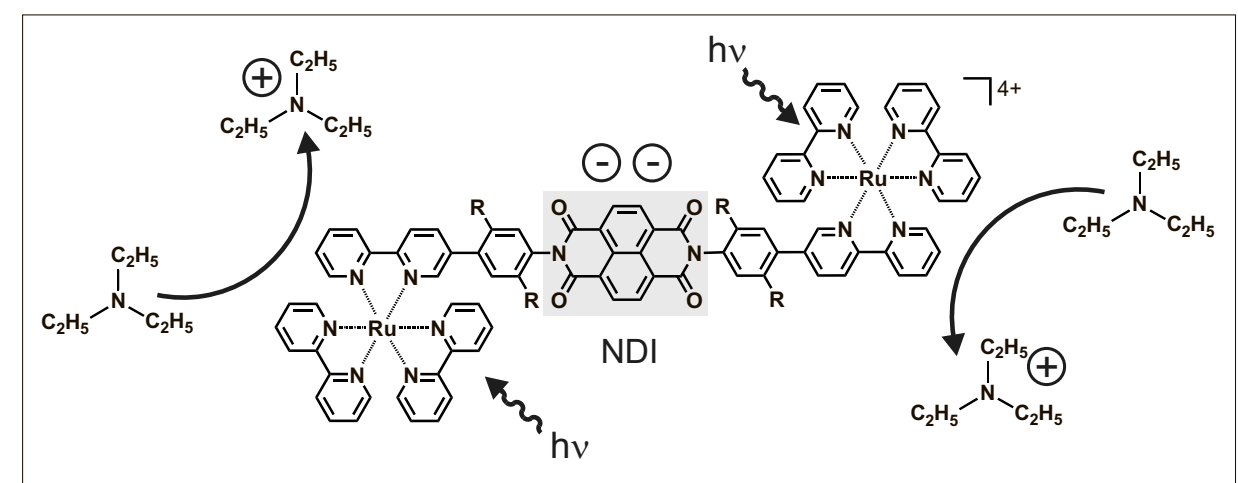

Fig. 2. Principle of photodriven charge accumulation on a central naphthalene diimide (NDI) unit in a molecular triad.

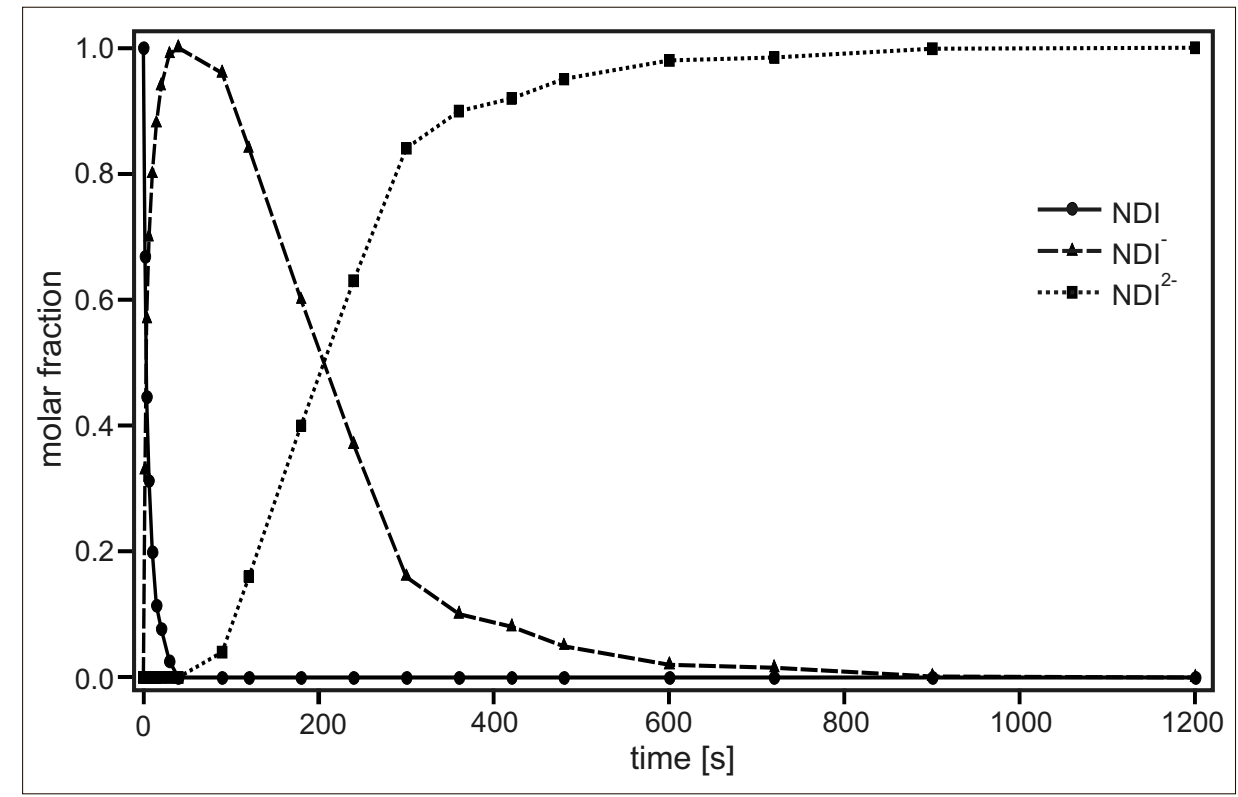

Fig. 3. Molar fractions of $\mathrm{NDI} \mathrm{NDI}^{-}$, and $\mathrm{NDI}^{2-}$ present after different irradiation times; see text for details. lective reduction reactions. ${ }^{[13]}$ With the aim of developing molecular factories incorporating both natural and artificial enzymes, we have demonstrated that such artificial metalloenzymes based on the biotin-streptavidin technology i) maintain their activity upon incorporation within artificial organelles $^{[14]}$ and ii) are fully compatible with a broad palette or natural enzymes. [1] We propose to engineer compartmentalized molecular factories consisting of an artificial photosynthetic reaction center, an artificial imine reductase, ${ }^{[13]}$ and an hemedependent monooxygenase ${ }^{[15]}$ for the production of high added value alkaloids (Fig. 4).

\section{Challenges Associated with Compartmentalization}

Similar to natural photosynthesis, the artificial photosynthetic reaction center in Fig. 1 will rely on establishing a redox gradient across a membrane using visible light as a driving-force. This requires proper 


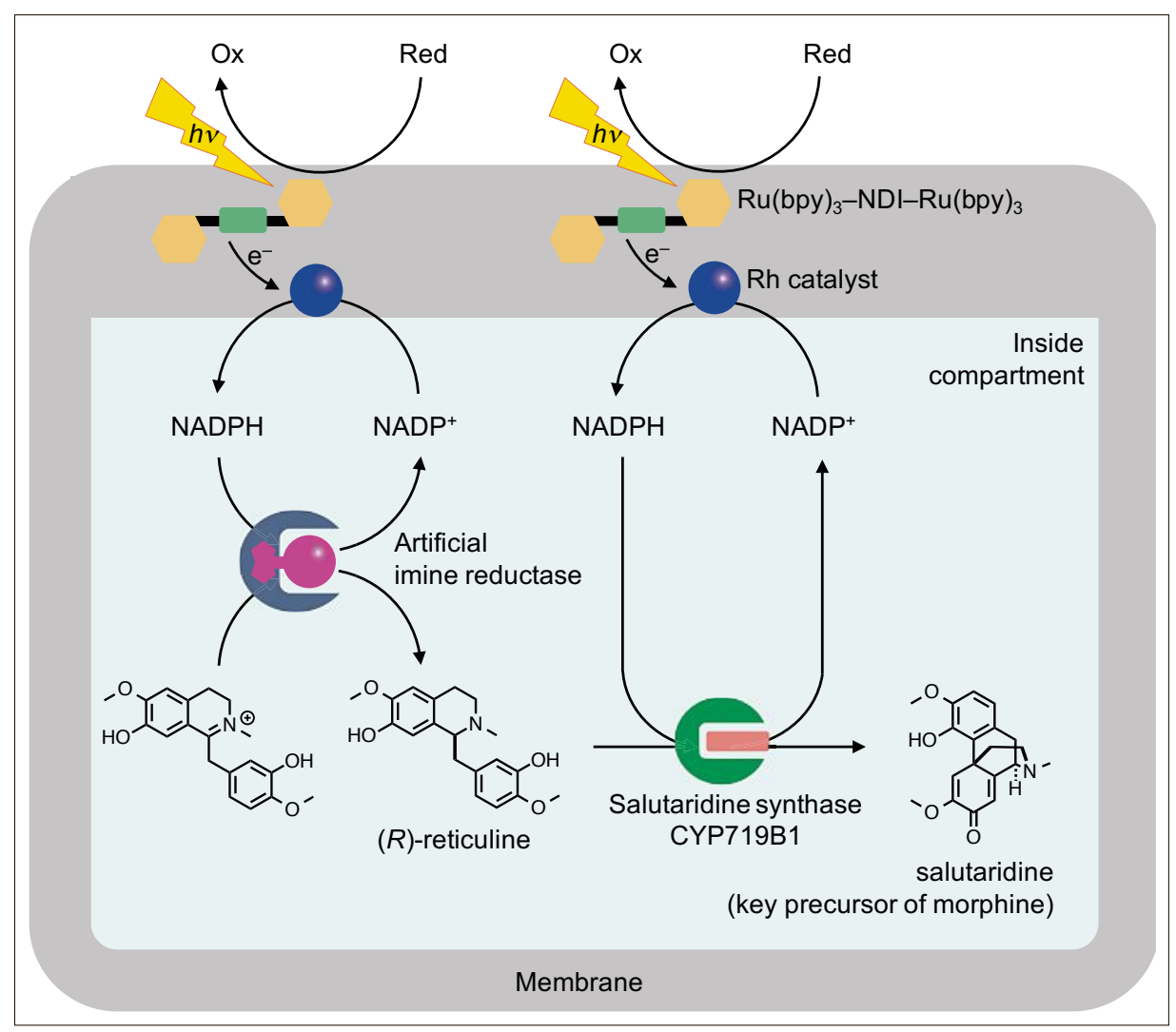

Fig. 4. Combining an artificial photosynthetic reaction center with an artificial imine reductase and salutaridine synthase affords an enzyme cascade for synthesis of a key morphine precursor.

orientation of the sub-components of this reaction center in the membrane, otherwise the net transfer of reduction equivalents to the inside of the compartment and their accumulation in the molecular factory will not be possible. For this purpose, we envision the use of asymmetric ABC triblock copolymer membranes, which are comprised of three chemically distinct polymer constituents such as for example poly(ethylene oxide), poly(dimethyl siloxane), and poly(methyl oxazoline).[16] Such ABC triblock copolymers are known to form vesicles that mimic biomembranes ${ }^{[17,18]}$ and they seem well suited for formation of closed compartments for molecular factories as proposed in Fig. 1.
Our strategy will be to exploit polarity differences between the individual block copolymer constituents to orient guest molecules (such as molecular wires) in the membrane. Recently, it has been demonstrated that membrane proteins can be oriented successfully in hybrids of amphiphilic block copolymers and lipids, ${ }^{[19]}$ and if molecular wires are equipped with suitable substituents at their periphery, preferential orientation of these wires in one direction should be achievable as well. This orientation will form the basis for net electron transfer from the outside to the inside compartment of the molecular factory, minimizing loss from photodriven charge transfer in the opposite direction.

\section{Acknowledgments}

Support from the Swiss National Science Foundation through the NCCR Molecular Systems Engineering is gratefully acknowledged.

Received: March 10, 2016

[1] V. Köhler, Y. M. Wilson, M. Durrenberger, D Ghislieri, E. Churakova, T. Quinto, L. Knörr, D. Häussinger, F. Hollmann, N. J. Turner, T. R Ward, Nat. Chem. 2013, 5, 93.

[2] M. Mifsud, S. Gargiulo, S. Iborra, I. Arends, F Hollmann, A. Corma, Nat. Commun. 2014, 5, 3145.

[3] C. M. Thomas, T. R. Ward, Chem. Soc. Rev. 2005, 34, 337.

[4] T. R. Ward, Acc. Chem. Res. 2011, 44, 47.

[5] A. Fihri, V. Artero, M. Razavet, C. Baffert, W. Leibl, M. Fontecave, Angew. Chem. Int. Ed. 2008, 47, 564.

[6] J. L. Dempsey, B. S. Brunschwig, J. R. Winkler, H. B. Gray, Acc. Chem. Res. 2009, 42, 1995.

[7] S. Berardi, S. Drouet, L. Francas, C. GimbertSurinach, M. Guttentag, C. Richmond, T. Stoll, A. Llobet, Chem. Soc. Rev. 2014, 43, 7501.

[8] A. G. Bonn, M. Neuburger, O. S. Wenger, Inorg. Chem. 2014, 53, 11075.

[9] A. G. Bonn, O. Yushchenko, E. Vauthey, O. S Wenger, Inorg. Chem. 2016, 55, 2894.

[10] M. Skaisgirski, X. Guo, O. S. Wenger, manuscript in preparation.

[11] K. T. Oppelt, E. Woss, M. Stiftinger, W. Schofberger, W. Buchberger, G. Knör, Inorg. Chem. 2013, 52, 11910.

[12] T. Ghosh, T. Slanina, B. König, Chem. Sci. 2015, 6, 2027.

[13] Y. Okamoto, V. Köhler, T. R. Ward, J. Am. Chem. Soc. 2016, 138, 5781.

[14] T. Heinisch, K. Langowska, P. Tanner, J. L. Reymond, W. Meier, C. Palivan, T. R. Ward, ChemCatChem 2013, 5, 720.

[15] S. C. Farrow, J. M. Hagel, G. A. W. Beaudoin, D. C. Burns, P. J. Facchini, Nat. Chem. Biol. 2015, 11, 728 .

[16] R. Stoenescu, W. Meier, Chem. Commun. 2002, 3016.

[17] K. Kita-Tokarczyk, J. Grumelard, T. Haefele, W. Meier, Polymer 2005, 46, 3540.

[18] C. G. Palivan, R. Goers, A. Najer, X. Y. Zhang, A. Car, W. Meier, Chem. Soc. Rev. 2016, 45, 377.

[19] J. Kowal, D. L. Wu, V. Mikhalevich, C. G Palivan, W. Meier, Langmuir 2015, 31, 4868. 